\title{
Expression of brain-specific angiogenesis inhibitor 1 is inversely correlated with pathological grade, angiogenesis and peritumoral brain edema in human astrocytomas
}

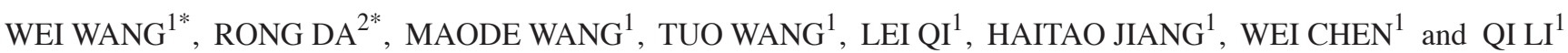 \\ ${ }^{1}$ Department of Neurosurgery and ${ }^{2}$ Clinical Laboratory, \\ The First Affiliated Hospital of the Medical College of Xi'an Jiaotong University, Xi'an, Shaanxi 710061, P.R. China
}

Received December 2, 2012; Accepted February 28, 2013

DOI: $10.3892 / 01.2013 .1250$

\begin{abstract}
As the most common intracranial malignant neoplasms, astrocytomas are characterized by high neovascularization and severe peritumoral brain edema (PTBE). Angiogenesis is a prerequisite for the growth of solid tumors, including astrocytoma, and brain-specific angiogenesis inhibitor 1 (BAI1) is a novel angiogenesis inhibitor. In the present study, the expression levels of BAI1, vascular endothelial growth factor (VEGF) and basic fibroblast growth factor (bFGF) were investigated using immunohistochemical methods in 90 human brain astrocytoma specimens of various pathological grades and in 11 normal human brain tissues. Vascular endothelial cells were stained for CD105 and the microvessel density (MVD) was assessed. The volume of astrocytoma and PTBE in each case was evaluated by magnetic resonance imaging (MRI). The results showed that BAI1 was highly expressed in the normal brain tissues, but that the expression decreased with the rising pathological grades of astrocytoma, MVD number and PTBE, indicating that BAI1 expression was inversely correlated with these factors. Furthermore, it was observed that the expression of VEGF and bFGF were inversely correlated with BAI1 expression in the human brain astrocytomas. These results indicate that the BAI1 gene may be used as a marker of decreased tumor progression and tumoral neovascularization, as well as PTBE.
\end{abstract}

Correspondence to: Professor Maode Wang, Department of Neurosurgery, The First Affiliated Hospital of the Medical College of Xi'an Jiaotong University, 277 Yanta West Road, Xi'an, Shaanxi 710061, P.R. China

E-mail: maodewang@yahoo.com.cn

${ }^{*}$ Contributed equally

Key words: brain-specific angiogenesis inhibitor 1, angiogenesis, peritumoral brain edema, astrocytoma

\section{Introduction}

Astrocytoma is the most common intracranial neoplasm and high-grade astrocytoma is characterized by high neovascularization. In high-grade gliomas, endothelial cell proliferation is 40-fold greater than that of normal brain tissue (1). Tumor vessels are characterized by an increased vessel diameter, length, density and permeability. It has been revealed that the development and growth of the tumor neovasculature are tightly regulated by $>19$ angiogenic factors and 300 antiangiogenic factors (2). Furthermore, the increased levels of angiogenic factors and tumor vessels in astrocytoma lead to severe perilesional edema, while vascular normalization substantially alleviates brain edema in patients with glioblastoma $(3,4)$. Pope et al observed that brain edema evaluated by magnetic resonance imaging (MRI) was an independent prognostic factor in patients with malignant gliomas and that patients with gliomas accompanied by severe brain edema often experienced poor clinical outcomes (5).

A novel antiangiogenic gene family, which includes three homologous genes, has been isolated and designated as the brain-specific angiogenesis inhibitor (BAI) family, consisting of BAI1, 2 and 3. BAI1 was isolated from p53 target genes and observed to be expressed specifically in the brain tissue, although this expression was absent in the majority of human glioma cell lines and downregulated in metastatic brain tumors from primary lung adenocarcinoma, indicating that BAI1 may be a tumor suppressor gene for intracranial neoplasms $(6,7)$. BAI1 is a p53 target gene encoding a 1,584-amino acid protein, which is expressed specifically in the brain. Nishimori et al observed that wild-type p53 induced the transcription of BAI1, a membrane protein composed of a seven-span transmembrane region and an extracellular domain with five TSP-type 1 repeats which form the functional antiangiogenesis domain of BAI1 (7). Nishimori et al demonstrated that BAI1 suppressed the angiogenesis induced by basic fibroblast growth factor (bFGF) in rat corneas using the corneal pocket assay (7). However, the correlation between BAI1 and bFGF and whether the suppression exists in human gliomas remains unknown.

In the present study, the expression of BAI1 was evaluated in normal human brain tissues and astrocytoma specimens of 
various grades, and then the correlation between BAI1 expression and microvessel density (MVD) labeled with CD105 (endoglin) was analyzed to investigate whether BAI1 may be used as a marker for angiogenesis in astrocytomas. A correlation was identified between BAI1 expression and the expression of two potent angiogenesis factors, vascular endothelial growth factor (VEGF) and bFGF. Immunohistochemistry was used to detect the expression of BAI1, VEGF and bFGF. The experiments were performed using standardized procedures to ensure that the results of the immunohistochemistry could be assessed semiquantitatively (8). The possibility of using BAI1 as a marker for peritumoral brain edema (PTBE) in astrocytomas was also evaluated.

\section{Materials and methods}

Patients, specimens and tissue preparation. The study cohort consisted of 90 patients with brain astrocytomas, who underwent surgical resection of the tumors at the First Affiliated Hospital of the Medical College of Xi'an Jiaotong University (Xi'an, China) between January 2008 and August 2010. The study was approved by the Ethics Committee of the First Affiliated Hospital of the Medical College of Xi'an Jiaotong University, Xi'an, China. All specimens were obtained from supratentorial surgical resection and divided into four groups according to the World Health Organization (WHO) classification of brain tumors (9) as follows: grade I, 21 cases; grade II, 24 cases; grade III, 27 cases; and grade IV, 18 cases. In total, 11 normal brain specimens, including the cortex and white matter, were obtained at autopsy from patients without any evidence of brain tumors. All patients' families provided written informed consent prior to enrolment. Within $10 \mathrm{~min}$ of surgical resection, the tissues were fixed with freshly prepared $10 \%$ formalin at $4^{\circ} \mathrm{C}$ for $24 \mathrm{~h}$ and embedded in paraffin. A pathologist reviewed the astrocytoma specimens to make the pathological diagnoses.

Immunohistochemistry. The formalin-fixed,paraffin-embedded tissues were serially sectioned to $5-\mu \mathrm{m}$ thick and collected on poly-L-lysine-coated slides. Staining was performed using immunoperoxidase-staining kits for mouse and rabbit antibodies using goat immunoglobulin.

Following routine deparaffinization and rehydration, the tissue sections were incubated with $3.0 \%$ hydrogen peroxide at room temperature (RT) for $10 \mathrm{~min}$ to block the endogenous peroxidase. The slides were placed in a glass jar filled with $10 \mathrm{mM}$ sodium citrate buffer ( $\mathrm{pH} \mathrm{6.0)}$ and boiled for 25 min within a microwave oven to retrieve the antigens. The slides were then incubated with $5 \%$ bovine serum albumin (BSA) at RT for 20 min to reduce the non-specific binding. The sections were incubated with the following primary antibodies: rabbit anti-BAI1 affinity purified polyclonal antibody (AB9364, 1:1,000; Chemicon International, Temecula, CA, USA); CD105 (Endoglin) mouse monoclonal antibody (NCL-CD105, 1:100; Novocastra, Newcastle upon Tyne, UK); VEGF (C-1) mouse monoclonal antibody (sc-7269, 1:600; Santa Cruz Biotechnology Inc., Santa Cruz, CA, USA); bFGF (147) rabbit polyclonal antibody (sc-79, 1:800; Santa Cruz Biotechnology Inc., Santa Cruz, CA, USA). Subsequent to incubation with the primary antibodies at $4^{\circ} \mathrm{C}$ overnight, the sections were rinsed with PBS and incubated with biotinylated goat anti-mouse immunoglobin (for VEGF and CD105), or biotinylated goat anti-rabbit immunoglobulin (for BAI1 and bFGF) at $37^{\circ} \mathrm{C}$ for $20 \mathrm{~min}$. The sections were then rinsed and incubated with avidin-biotin-horseradish peroxidase complex at $37^{\circ} \mathrm{C}$ for $20 \mathrm{~min}$. The sections were visualized with 3,3'-diaminobenzidine tetrahydrochloride and counterstained with hematoxylin.

Evaluation of immunohistochemical staining. Two pathologists, who were blinded to the pathological diagnoses and clinical data, observed the immunohistochemical staining results. A total of 1,000 cells in five random high-powered fields at x400 magnification were observed per slide and the immunopositive cells for BAI1, VEGF and bFGF were counted. Semiquantitative analysis was performed to determine the expression levels of BAI1, VEGF and bFGF. The labeling index (LI) was defined as the percentage of immunopositive cells (ratio of positively stained cells to the total number of cells per slide, multiplied by 100).

Identification of MVD by CD105 staining. With the CD105 staining, the MVD of each section was calculated according to Weidner's criteria (10). Briefly, each section was observed under low-powered fields (LPF) at x100 magnification to identify the 'hot spots'. The microvessels of each 'hot spot' were then counted in a x200 magnification field. Single endothelial cells or the clusters of endothelial cells, which were stained dark brown and clearly separated from adjacent astrocytoma cells or connective tissues, were recognized as countable microvessels. A total of five randomly selected fields at x200 magnification per slide were evaluated and the mean MVD was calculated.

Assessment of the astrocytoma volume, PTBE and edema index (EI) by MRI. The astrocytoma volume and PTBE of each case were evaluated by MRI (11). The volume of the tumor $\left(\mathrm{V}_{\text {tumor }}\right)$ was assessed by gadolinium-diethylenetriamine pentaacetic acid (Gd-DTPA)-enhanced T1-weighted imaging and the volume of the PTBE $\left(\mathrm{V}_{\text {edema }}\right)$ was evaluated by T2-weighted or fluid-attenuated inversion-recovery (FLAIR) imaging. In each case, the maximum coronal (a), axial (b) and sagittal (c) radii, which were perpendicular to each other, were measured. The tumor and PTBE volumes were calculated according to the following formula:

$$
V=4 / 3 \pi a b c
$$

The correlation between the tumor and PTBE volumes in each case was defined as the EI:

$$
E I=\frac{V_{\text {iumor }}+V_{\text {edema }}}{V_{\text {tumor }}}
$$

Statistical analysis. The SPSS 13.0 software package was used for all statistical analyses. The data are presented as the mean \pm standard error. Differences in the MVD, EI and LI of BAI1, VEGF and bFGF were analyzed among the groups by one-way ANOVA. The correlations between the MVD (or EI) and the LI of BAI1, VEGF and bFGF were analyzed 

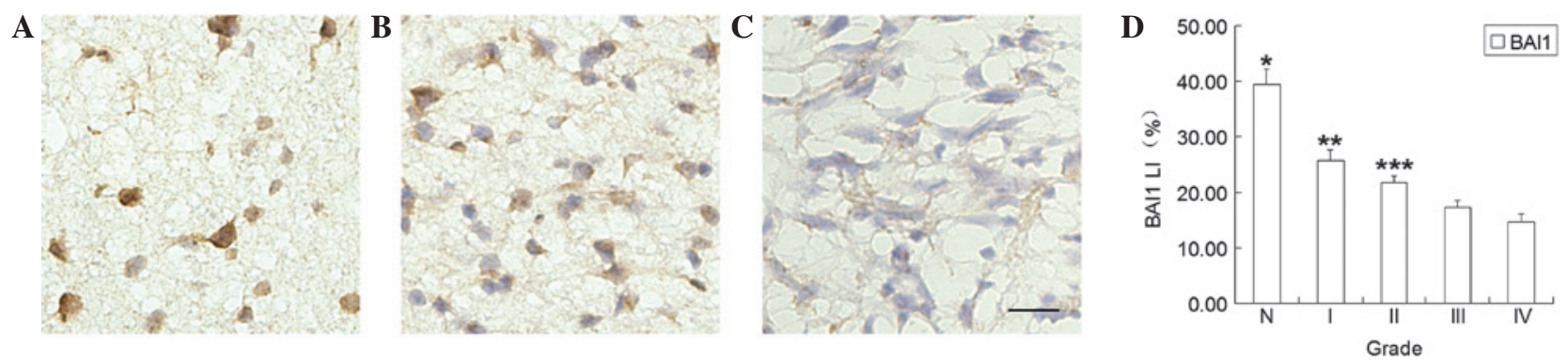

Figure 1. Expression of BAI1 in normal brain tissue and astrocytoma specimens of various grades. The expression of BAI1 was higher in (A) normal brain tissues than (B) low-grade astrocytomas and (C) high-grade astrocytomas. BAI1 expression was higher in (B) low-grade astrocytomas than (C) high-grade astrocytomas. Scale bar $=30 \mu \mathrm{m}$. (D) The LI of BAI1 decreased with the pathological grade of the astrocytoma (Spearman's rank correlation, $\mathrm{r}=-0.519$, $\mathrm{P}<0.01)$. " $\mathrm{P}<0.05$ vs. grade I, II, III and IV astrocytomas, ${ }^{* *} \mathrm{P}<0.05$ vs. grades III and IV, ${ }^{* * * *} \mathrm{P}<0.05$ vs. grade IV. BAI1, brain-specific angiogenesis inhibitor 1 ; LI, labeling index; N, normal brain tissue.

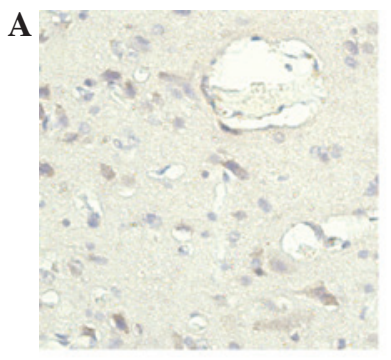

D

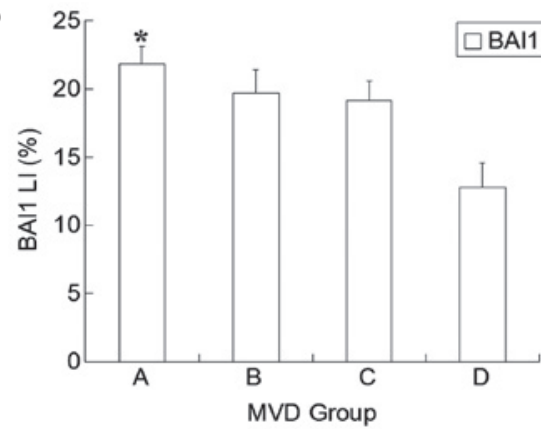

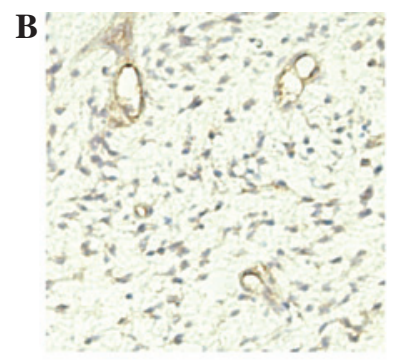
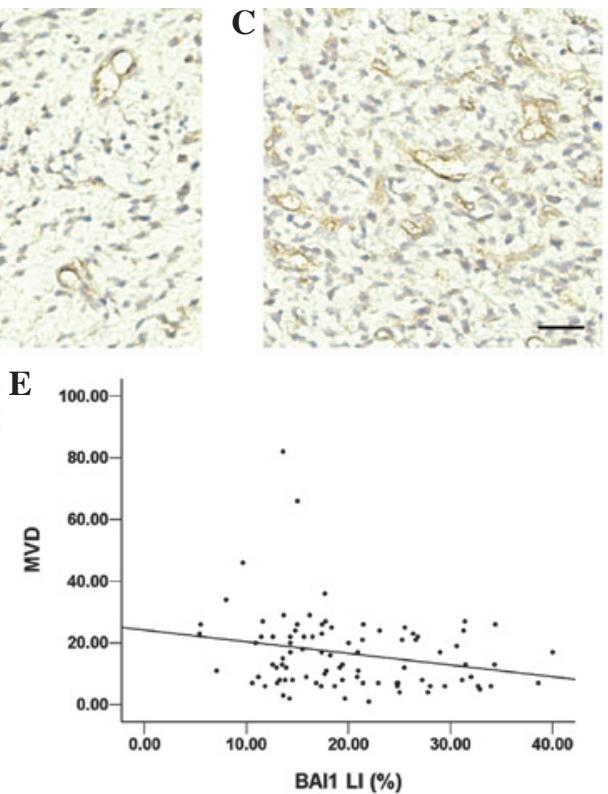

Figure 2. MVD in normal brain tissue and various grades of astrocytomas. MVD was lower in (A) normal brain tissues compared with (B) low-grade and (C) high-grade astrocytomas and higher in (C) high-grade astrocytomas than (B) low-grade astrocytomas. Scale bar $=30 \mu \mathrm{m}$. (D) Astrocytoma specimens were divided into four groups according to their MVD value: group A, MVD $\leq 10$; group B, $10<$ MVD $\leq 20$; group C, 20< MVD $\leq 30$; group D, MVD $>30$. ${ }^{*} \mathrm{P}<0.05$ vs. group D. (E) The MVD value of the astrocytomas decreased with increasing BAI1 expression (Spearman's rank correlation, $r=-0.222, \mathrm{P}<0.05$ ). MVD, microvessel density; BAI1, brain-specific angiogenesis inhibitor 1.

by a Spearman's rank correlation. $\mathrm{P}<0.05$ was considered to indicate a statistically significant difference.

\section{Results}

Decreased expression of BAII in human astrocytomas of various grades. Immunohistochemical staining showed that BAI1 was expressed in the cytoplasm of normal human astrocytes and neurons, but only in few human brain astrocytoma cells. The BAI1 expression was high in the normal brain tissues, moderate in the low-grade astrocytomas and low in the high-grade astrocytomas (Fig. 1A-C). There were significant differences between the normal brain tissue and the astrocytomas of the various grades, as well as between grade I and grades III and IV, and grade II and grade IV $(\mathrm{P}<0.05$; Fig. 1D). The BAI1 immunostaining in the astrocytomas decreased with the increasing tumor grades (Fig. 1A-C), and the BAI1 LI was inversely correlated with the pathological grade of the astrocytomas (Spearman's rank correlation, $\mathrm{r}=-0.519, \mathrm{P}<0.01)$.

BAII expression is negatively correlated with MVD in human astrocytomas. Endothelial cells stained for CD105 were more abundant in the astrocytoma specimens compared with the normal brain tissue. The MVD in the normal brain tissue was significantly lower than that in the astrocytomas (Fig. 2A-C). In the astrocytoma specimens, the MVD decreased as the BAI1 LI increased. To evaluate the correlation between BAI1 and MVD, all astrocytoma specimens were divided into four groups according to their MVD values: group A, MVD $\leq 10$; group $B, 10<$ MVD $\leq 20$; group $\mathrm{C}, 20<\mathrm{MVD} \leq 30$; and group $\mathrm{D}, \mathrm{MVD}>30$. The BAI1 expression in group A was greater than in group $\mathrm{D}(\mathrm{P}<0.05$; Fig. 2D). A negative correlation was observed between MVD and BAI1 LI expression (Spearman's rank correlation, $\mathrm{r}=-0.222, \mathrm{P}<0.05$; Fig. 2E). 

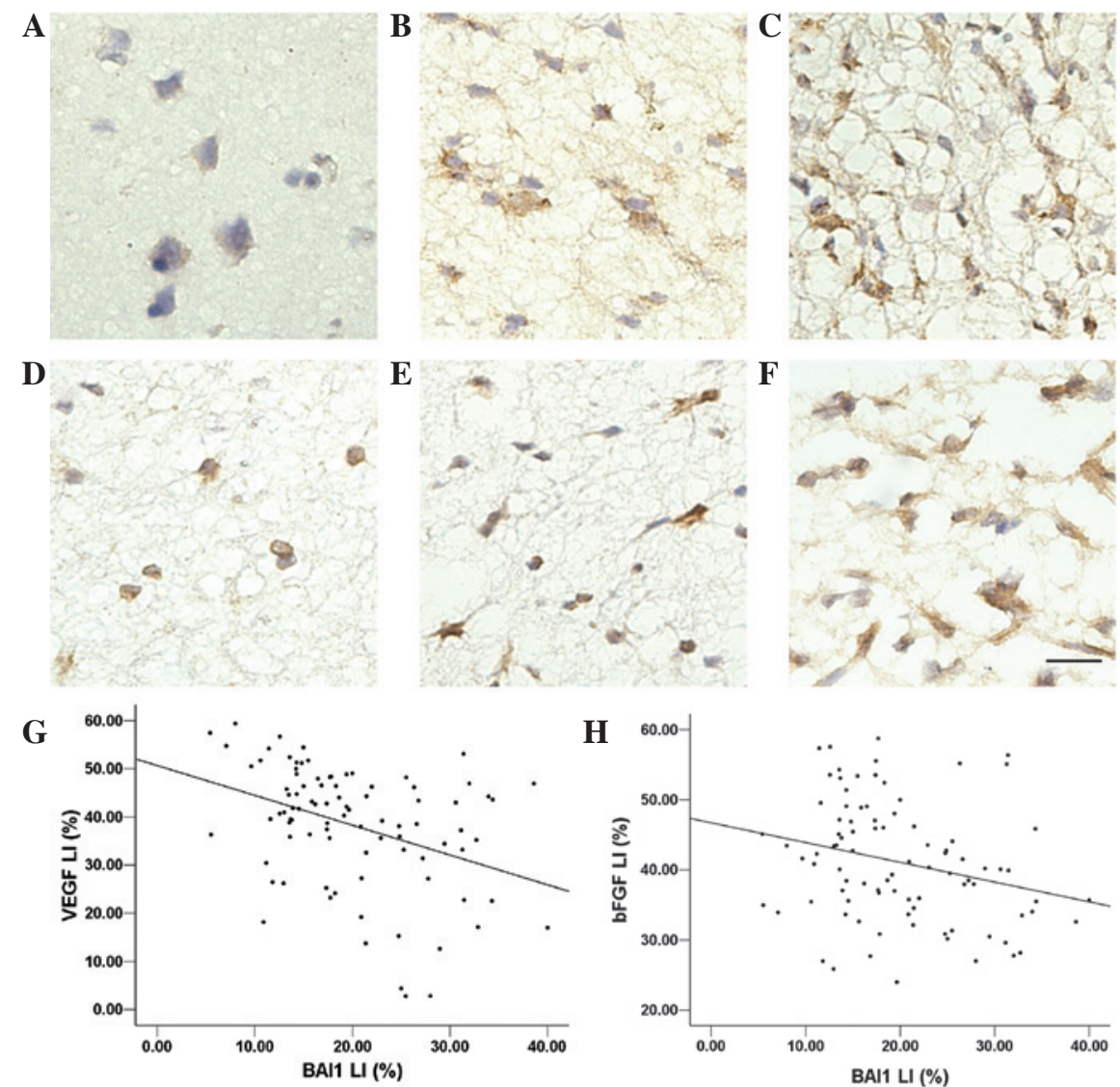

Figure 3. Expression of VEGF was lower in (A) normal brain tissues than in (B) low-grade and (C) high-grade astrocytomas and higher in (C) high-grade astrocytomas than (B) low-grade astrocytomas. Expression of bFGF was lower in (D) normal brain tissues than in (E) low-grade and (F) high-grade astrocytomas and higher in (F) high-grade astrocytomas than (E) low-grade astrocytomas. (G) VEGF expression in astrocytomas decreased with increased BAI1 expression (Spearman's rank correlation, $\mathrm{r}=-0.379, \mathrm{P}<0.01)$. $(\mathrm{H})$ bFGF expression in astrocytomas decreased with increased BAI1 expression (Spearman's rank correlation, $\mathrm{r}=-0.277, \mathrm{P}<0.01$ ). BAI1, brain-specific angiogenesis inhibitor 1; VEGF, vascular endothelial growth factor; bFGF, basic fibroblast growth factor; LI, labeling index.
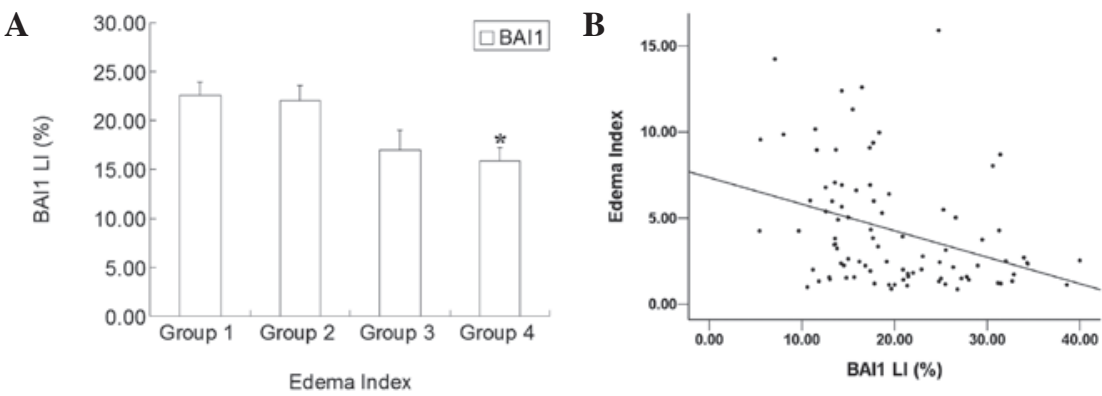

Fig 4. (A) BAI1 expression in astrocytomas was different in patients with various EIs. Astrocytoma specimens were divided into four groups according to their EI: group 1, EI $\leq 2$; group 2, $2<\mathrm{EI} \leq 4$; group $3,4<\mathrm{EI} \leq 6$; group 4, EI $>6$. ${ }^{*} \mathrm{P}<0.05$ vs. groups 1 and 2 . (B) PTBE in astrocytomas decreased with increased BAI1 expression (Spearman's rank correlation, $\mathrm{r}=-0.380, \mathrm{P}<0.01$ ). BAI1, brain-specific angiogenesis inhibitor 1; EI, edema index; PTBE, peritumoral brain edema.

BAIl expression is negatively correlated with the expression of VEGF and bFGF in astrocytomas. The BAI1 expression levels were inversely correlated with the tumor neovasculature in the astrocytomas. Furthermore, the correlations between BAI1 expression and two potent angiogenic factors, VEGF and bFGF, were also evaluated. Positive immunostaining for VEGF was observed in the cytoplasm of the endothelial and astrocytoma cells and in small amounts in the astrocytic cells. Normal brain tissue seldomly expressed VEGF, while mild staining was observed in the low-grade astrocytomas and marked expression was observed in the high-grade astrocy- tomas (Fig. 3A-C). In the astrocytoma tissues, the VEGF LI decreased as the BAI1 LI increased. VEGF expression was negatively correlated with BAI1 expression (Spearman's rank correlation, r=-0.379, $\mathrm{P}<0.01$; Fig. 3G).

Positive staining for bFGF was observed in the cytoplasm of the astrocytoma, endothelial and astrocytic cells. The expression of bFGF was stronger in the high-grade astrocytomas than the low-grade cytomas and markedly stronger than in the normal brain tissue (Fig. 3D-F). In the astrocytoma specimens, the bFGF LI decreased as the BAI1 LI increased. The expression of bFGF was negatively correlated with BAI1 
expression (Spearman's rank correlation, $\mathrm{r}=-0.277, \mathrm{P}<0.01$; Fig. 3H).

BAIl expression is negatively correlated with PTBE in astrocytomas. Tumoral vessels are characterized by high permeability, which results in severe PTBE. The BAI1 expression was inversely correlated with the tumor neovasculature in the astrocytomas, therefore, its correlation with the PTBE level was investigated. The astrocytoma specimens were divided into four groups according to the EI: group 1, EI $\leq 2$; group 2, $2<\mathrm{EI} \leq 4$; group 3, $4<\mathrm{EI} \leq 6$; and group $4, \mathrm{EI}>6$. The BAI1 LI of group 4 was significantly lower than groups 1 and $2(\mathrm{P}<0.05$; Fig. 4A). The BAI1 expression decreased with the increasing PTBE and was inversely correlated with the EI (Spearman's rank correlation, r=-0.380, P<0.01; Fig. 4B).

\section{Discussion}

The growth of tumors relies on the formation of their own vascular network (neovascularization), which provides nutrition and oxygen and removes carbon dioxide and other metabolic waste. High levels of vascularization, which contribute to rapid tumor growth and severe PTBE, have been observed in the majority of astrocytomas, particularly in malignant astrocytomas (4). Angiogenesis and antiangiogenesis factors modulate the neovascularization of astrocytomas and also affect PTBE (18). In the present study, the correlations between a novel antiagiogenic factor, BAIl, and vascularization and peritumoral edema were investigated in human brain astrocytomas.

The present results showed that BAIl was highly expressed in the cytoplasm of astrocytes and neurons in the normal brain tissues, while the expression decreased significantly with the increasing pathological grade in the human astrocytoma specimens. The expression of BAI1 was rare in high-grade astrocytoma specimens, particularly grade IV. These results were consistent with in vitro studies, which showed that BAI1 mRNA and protein were absent from the majority of human glioma cell lines $(7,12)$. Izutsu et al demonstrated that BAI1 mRNA and protein were downregulated in advanced renal cell carcinoma compared with localized renal cell carcinoma, indicating that BAI1 was crucial for renal cell carcinoma development (13). In addition, BAI1 was observed to be downregulated in pulmonary adenocarcinomas and gastric and colorectal cancers compared with normal tissues (14-17). BAI1 is inversely correlated with the pathological grade of the astrocytoma and may be used as a marker of decreasing malignancy for astrocytomas and other malignant neoplasms.

The MVD was investigated in the human astrocytomas and normal brain tissue and its correlation with BAI1 expression was analyzed. It has been demonstrated that MVD labeled with CD105, which has specific affinity for activated endothelial cells, is a better prognostic factor than either CD31 or CD34 (18-21). Therefore, CD105 was used in the present study as a biomarker for determining the MVD of human astrocytoma specimens and normal human brain tissue. The present study showed that the MVD labeled with CD105 was inversely correlated with BAI1 expression, indicating that BAI1 may be used as a marker of decreased tumoral neovascularization in human astrocytomas.
Other studies have investigated the function of BAI1 in tumoral angiogenesis in vitro and in vivo and revealed that ectogenic BAI1 suppresses the vascularization of gliomas, pancreatic cancers and renal cell carcinomas (15,22-25). An in vivo study showed that an adenoviral vector encoding BAI1-transduced glioblastoma cell xenografts exhibited significant suppression of tumor growth and impairment of tumor angiogenesis (36). However, the antiangiogenic mechanism of BAI1 in human astrocytomas has not been thoroughly investigated. The BAI1 extracellular domain may be separately proteolyzed and cleaved into $120-\mathrm{kDa}$ [Vasculostatin-120 (Vstat120)] and 40-kDa [Vasculostatin-40 (Vstat40)] fragments, which have significant antiangiogenic activities in vitro and in vivo, depending on the presence of the $\mathrm{CD} 36$ receptor on the target endothelial cells (26-28). Moreover, BAI1 has been shown to specifically inhibit endothelial cell migration, which also contributes to the antiangiogenic properties of BAI1 (16).

The balance between angiogenic and antiangiogenic factors is crucial for the clinical prognosis of patients with glioblastomas. VEGF and bFGF are two potent angiogenic factors. Kudo et al noted that BAI1 inhibited the angiogenesis of murine renal cell carcinoma by suppressing VEGF expression (23). Nishimori et al observed that the neovascularization induced by bFGF was inhibited by BAI1 (7). Nam et al demonstrated that patients with glioblastomas with high VEGF and absent BAI1 mRNA expression often experienced poor outcomes (22).

Furthermore, the correlations between the expression levels of BAI1 and two potent angiogenic factors, VEGF and bFGF, were analyzed using the astrocytoma specimens. Despite the degrees of malignancy, the present study showed that the lower the BAI1 expression, the higher the VEGF and bFGF expression levels. Whether the BAI1 expression inhibited the expression of VEGF and bFGF, or vice versa, requires further investigation in the future.

Severe astrocytoma PTBE induces elevated intracranial pressure and leads to poor clinical outcomes. Patients with glioblastoma multiforme without PTBE experienced double the median survival time compared with those with PTBE (5). Higher tumor angiogenesis and vasogenic edema have also been associated with higher morbidity and mortality rates in patients with malignant brain tumors (3).

The present study showed that BAI1 expression was inversely correlated with PTBE in human brain astrocytomas, indicating that BAI1 may be used as a marker of decreased PTBE for patients with this condition. Schoenegger et al observed that patients with major edema experienced significantly shorter overall survival times compared with patients with minor edema (29). PTBE was likely to affect morbidity and the invasive potential of malignant gliomas (30). Therefore, patients with higher BAI1 expression may experience minor PTBE and superior overall survival.

BAI1 is a multi-functional protein with crucial roles in cell adhesion and signal transduction in the brain (31). BAI1 has the ability to mediate membrane-cytoskeletal interactions and signal transduction during neuronal growth (32) and also to regulate neurotransmitter release (33). BAI1 is critical for neural development, synapse formation, signal transduction at neuron synapses, cell proliferation and cytokinesis (34). Koh et al revealed that BAI1 inhibited neuronal differentia- 
tion (35). A clinical study showed that patients with gliomas expressing BAI1 were sensitive to radiotherapy, suggesting that exogenous BAI1 may increase the sensitivity of astrocytomas to radiation (22).

In summary, BAI1 is inversely correlated with pathological grade, angiogenesis and PTBE and may be used as a marker for decreasing malignancy, neovascularization and perilesional brain edema in human astrocytomas. Further research focused on the function of BAI1 should be performed in the future.

\section{Acknowledgements}

The authors would like to thank Peilong Cao for the pathological diagnoses and Kai Wang and Changan Zhao for the observation of immunohistochemical staining (Peilong Cao and Kai Wang, Department of Pathology, The First Affiliated Hospital of the Medical College of Xi'an Jiaotong University; Changan Zhao, Department of Pathology, Medical College of Xi'an Jiaotong University, China). The present study was supported by the National Natural Science Foundation of China (grant no. 30772226) and the Fundamental Research Fund of Xi'an Jiaotong University.

\section{References}

1. Plate KH: Mechanisms of angiogenesis in the brain. J Neuropathol Exp Neurol 58: 313-320, 1999.

2. Harrigan MR: Angiogenic factors in the central nervous system. Neurosurgery 53: 639-661, 2003.

3. Batchelor TT, Sorensen AG, di Tomaso E, et al: AZD2171, a pan-VEGF receptor tyrosine kinase inhibitor, normalizes tumor vasculature and alleviates edema in glioblastoma patients. Cancer Cell 11: 83-95, 2007.

4. Stiver SI: Angiogenesis and its role in the behavior of astrocytic brain tumors. Front Biosci 9: 3105-3123, 2004.

5. Pope WB, Sayre J, Perlina A, Villablanca JP, Mischel PS and Cloughesy TF: MR imaging correlates of survival in patients with high-grade gliomas. AJNR Am J Neuroradiol 26: 2466-2474, 2005 .

6. Zohrabian VM, Nandu H, Gulati N, et al: Gene expression profiling of metastatic brain cancer. Oncol Rep 18: 321-328, 2007.

7. Nishimori H, Shiratsuchi T, Urano T, et al: A novel brain-specific p53-target gene, BAI1, containing thrombospondin type 1 repeats inhibits experimental angiogenesis. Oncogene 15: 2145-2150, 1997.

8. Taylor CR and Levenson RM: Quantification of immunohistochemistry - issues concerning methods, utility and semiquantitative assessment II. Histopathology 49: 411-424, 2006.

9. Louis DN, Ohgaki H, Wiestler OD, et al: The 2007 WHO classification of tumours of the central nervous system. Acta Neuropathol 114: 97-109, 2007.

10. Weidner N, Semple JP, Welch WR and Folkman J: Tumor angiogenesis and metastasis - correlation in invasive breast carcinoma N Engl J Med 324: 1-8, 1991.

11. Oshio K, Binder DK, Liang Y, et al: Expression of the aquaporin-1 water channel in human glial tumors. Neurosurgery 56: 375-381, 2005.

12. Kaur B, Brat DJ, Calkins CC and Van Meir EG: Brain angiogenesis inhibitor 1 is differentially expressed in normal brain and glioblastoma independently of p53 expression. Am J Pathol 162: 19-27, 2003.

13. Izutsu T, Konda R, Sugimura J, Iwasaki K and Fujioka T: Brain-specific angiogenesis inhibitor 1 is a putative factor for inhibition of neovascular formation in renal cell carcinoma. J Urol 185: 2353-2358, 2011.

14. Hatanaka H, Oshika Y, Abe Y, et al: Vascularization is decreased in pulmonary adenocarcinoma expressing brain-specific angiogenesis inhibitor 1 (BAI1). Int J Mol Med 5: 181-183, 2000.

15. Fukushima Y, Oshika Y, Tsuchida T, et al: Brain-specific angiogenesis inhibitor 1 expression is inversely correlated with vascularity and distant metastasis of colorectal cancer. Int $\mathbf{J}$ Oncol 13: 967-970, 1998.
16. Miyamoto N, Yamamoto H, Taniguchi H, et al: Differential expression of angiogenesis-related genes in human gastric cancers with and those without high-frequency microsatellite instability. Cancer Lett 254: 42-53, 2007.

17. Yoshida Y, Oshika Y, Fukushima Y, et al: Expression of angiostatic factors in colorectal cancer. Int J Oncol 15: 1221-1225, 1999.

18. Yao Y, Kubota T, Sato K, Kitai R, Takeuchi H and Arishima H: Prognostic value of vascular endothelial growth factor and its receptors Flt-1 and Flk-1 in astrocytic tumours. Acta Neurochir (Wien) 143: 159-166, 2001.

19. Akagi K, Ikeda Y, Sumiyoshi Y, et al: Estimation of angiogenesis with anti-CD105 immunostaining in the process of colorectal cancer development. Surgery 131 (1 Suppl): S109-S113, 2002.

20. Behrem S, Zarkovic K, Eskinja N and Jonjic N: Endoglin is a better marker than CD31 in evaluation of angiogenesis in glioblastoma. Croat Med J 46: 417-422, 2005.

21. Mineo TC, Ambrogi V, Baldi A, et al: Prognostic impact of VEGF, CD31, CD34, and CD105 expression and tumour vessel invasion after radical surgery for IB-IIA non-small cell lung cancer. J Clin Pathol 57: 591-597, 2004.

22. Nam DH, Park K, Suh YL and Kim JH: Expression of VEGF and brain specific angiogenesis inhibitor-1 in glioblastoma: prognostic significance. Oncol Rep 11: 863-869, 2004.

23. Kudo S, Konda R, Obara W, et al: Inhibition of tumor growth through suppression of angiogenesis by brain-specific angiogenesis inhibitor 1 gene transfer in murine renal cell carcinoma. Oncol Rep 18: 785-791, 2007.

24. Xiao XR, Kang XX and Zhao JZ: Therapeutic effect of brain-specific angiogenesis inhibitor 1 on glioblastoma: an animal experiment. Zhonghua Yi Xue Za Zhi 86: 1342-1346, 2006 (In Chinese).

25. Yoon KC, Ahn KY, Lee JH, et al: Lipid-mediated delivery of brain-specific angiogenesis inhibitor 1 gene reduces corneal neovascularization in an in vivo rabbit model. Gene Ther 12: 617-624, 2005.

26. Kaur B, Brat DJ, Devi NS and Van Meir EG: Vasculostatin, a proteolytic fragment of brain angiogenesis inhibitor 1, is an antiangiogenic and antitumorigenic factor. Oncogene 24: 3632-3642, 2005.

27. Kaur B, Cork SM, Sandberg EM, Devi NS, Zhang Z, Klenotic PA, Febbraio M, Shim H, Mao H, Tucker-Burden C, Silverstein RL, Brat DJ, Olson JJ and Van Meir EG: Vasculostatin inhibits intracranial glioma growth and negatively regulates in vivo angiogenesis through a CD36-dependent mechanism. Cancer Res 69: 1212-1220, 2009.

28. Cork SM and Van Meir EG: Emerging roles for the BAI1 protein family in the regulation of phagocytosis, synaptogenesis, neurovasculature, and tumor development. J Mol Med (Berl) 89: 743-752.

29. Schoenegger K, Oberndorfer S, Wuschitz B, et al: Peritumoral edema on MRI at initial diagnosis: an independent prognostic factor for glioblastoma? Eur J Neurol 16: 874-878, 2009.

30. Da Fonseca CO, Silva JT, Lins IR, et al: Correlation of tumor topography and peritumoral edema of recurrent malignant gliomas with therapeutic response to intranasal administration of perillyl alcohol. Invest New Drugs 27: 557-564, 2009.

31. Shiratsuchi T, Futamura M, Oda K, Nishimori H, Nakamura Y and Tokino T: Cloning and characterization of BAI-associated protein 1: a PDZ domain-containing protein that interacts with BAI1. Biochem Biophys Res Commun 247: 597-604, 1998.

32. Oda K, Shiratsuchi T, Nishimori H, et al: Identification of BAIAP2 (BAI-associated protein 2), a novel human homologue of hamster IRSp53, whose SH3 domain interacts with the cytoplasmic domain of BAI1. Cytogenet Cell Genet 84: 75-82, 1999.

33. Shiratsuchi $\mathrm{T}$, Oda $\mathrm{K}$, Nishimori $\mathrm{H}$, et al: Cloning and characterization of BAP3 (BAI-associated protein 3), a C2 domain-containing protein that interacts with BAI1. Biochem Biophys Res Commun 251: 158-165, 1998.

34. Mori K, Kanemura Y, Fujikawa H, et al: Brain-specific angiogenesis inhibitor 1 (BAI1) is expressed in human cerebral neuronal cells. Neurosci Res 43: 69-74, 2002.

35. Koh JT, Lee ZH, Ahn KY, et al: Characterization of mouse brain-specific angiogenesis inhibitor 1 (BAI1) and phytanoyl-CoA alpha-hydroxylase-associated protein 1, a novel BAI1-binding protein. Brain Res Mol Brain Res 87: 223-237, 2001.

36. Kang X, Xiao X, Harata M, et al: Antiangiogenic activity of BAI1 in vivo: implications for gene therapy of human glioblastomas. Cancer Gene Ther 13: 385-392, 2006. 